\title{
Le Syndrome des Anticorps Anti Phospholipides: Une Etiologie des Fausses Couches Laquelle on Ne Pense pas Souvent en Afrique Noire
}

\author{
N'dhatz Comoe Emeraude, \\ Packo Dieu-le-veut Saint-Cyr Sylvestre, \\ Nanho Danho Clotaire,
} Service d'Hématologie Clinique, Centre Hospitalier Universitaire de Yopougon, Abidjan, Côte d'Ivoire

Karama Issa,

Service de Gynécologie Obstétrique,

Centre Hospitalier Universitaire de Yopougon, Abidjan, Côte d'Ivoire

Boidy Kouakou,

Service d'Hématologie Clinique,

Centre Hospitalier Universitaire de Yopougon, Abidjan, Côte d'Ivoire

Diakité Mamady,

Service d'Hématologie Clinique, Hôpital National de DONKA, Guinée Conakry

\section{Mbelesso Pascal,}

Service de Neurologie, Centre Hospitalier Universitaire de l'Amitié Sinocentrafricaine de Bangui, République Centrafricaine

Koffi Kouassi Gustave,

Service d'Hématologie Clinique,

Centre Hospitalier Universitaire de Yopougon, Abidjan, Côte d'Ivoire

Doi:10.19044/esj.2019.v15n30p172 URL:http://dx.doi.org/10.19044/esj.2019.v15n30p172

\section{Resume}

Contexte : Le syndrome des anticorps antiphospholipides est l'une des étiologies des fausses couches rarement évoquée en première intention, sous diagnostiquée en Afrique Noire. Presentation de Cas : Les auteurs rapportent l'observation d'une femme de 36 ans, aux antécédents d'ulcère de la jambe gauche, suivie au service de gynéco-obstétrique pour des fausses couches à répétition totalisant cinq (5) épisodes. Le diagnostic initial était orienté vers les causes gynéco-obstétricales, lesquelles trois cerclages du col ont été réalisés avec échec. La survenue d'une pancytopénie qui était le motif majeur de consultation de la patiente a permis de porter le diagnostic de syndrome des anticorps antiphospholipides lupiques par un titrage significatif des anticorps 
antiphospholipides et des anticorps antinucléaires de spécifié anti DNA. Le traitement avec les corticoïdes, immunosuppresseurs, l'aspirine et l'héparine a permis une évolution favorable conduisait une 6è grossesse avec succès. Conclusion: $\mathrm{Ce}$ travail interpelle les praticiens à rechercher systématiquement le syndrome des anticorps antiphospholipides devant toutes fausses couches spontanées en vue d'un diagnostic et prise en charge précoce.

Mots-Cles: Anticorps anti phospholipide, Fausse couche, Afrique Noire 


\title{
The Antiphospholipid Antibody Syndrome: An Etiology of Recurrent Abortion that People Often Do Not Think About in Black Africa
}

\author{
N'dhatz Comoe Emeraude, \\ Packo Dieu-le-veut Saint-Cyr Sylvestre, \\ Nanho Danho Clotaire, \\ Service d'Hématologie Clinique, Centre Hospitalier \\ Universitaire de Yopougon, Abidjan, Côte d'Ivoire
}

Karama Issa,

Service de Gynécologie Obstétrique,

Centre Hospitalier Universitaire de Yopougon, Abidjan, Côte d'Ivoire

Boidy Kouakou,

Service d'Hématologie Clinique,

Centre Hospitalier Universitaire de Yopougon, Abidjan, Côte d'Ivoire

Diakité Mamady,

Service d'Hématologie Clinique, Hôpital National de DONKA, Guinée Conakry

\section{Mbelesso Pascal,}

Service de Neurologie, Centre Hospitalier Universitaire de l'Amitié Sinocentrafricaine de Bangui, République Centrafricaine

Koffi Kouassi Gustave,

Service d'Hématologie Clinique,

Centre Hospitalier Universitaire de Yopougon, Abidjan, Côte d'Ivoire

\begin{abstract}
Background: The antiphospholipid antibody syndrome (APL) was rarely evoked as abortion etiology in black Africa. Case Report: The authors reported a case of a 36-year-old female with a medical history of leg ulcer who presented five episodes of recurrent abortion. The diagnosis was oriented to obstetrical etiologies, and three uterine cervix cerclages were realized with failure. As a result of pancytopenia which was the main reason of consultation, the diagnosis was made with immunology test showing a high titer of antiphospholipid antibody and lupus anticoagulant. The patient was treated with corticosteroids, immunosuppressive agents, aspirin, and heparin. The 6th pregnancy was free from complications and led to the birth of a living child.
\end{abstract}


Conclusion: This case calls on practitioners to systematically search for the antiphospholipid antibody syndrome during the investigation of spontaneous miscarriages for diagnosis and early management.

Keywords: Antiphospholipid antibody, recurrent abortion, Black Africa

\section{Introduction}

Le syndrome des anticorps anti phospholipides (SAPL) est un état de thrombophilie acquise, du à l'action des auto-anticorps dirigés contre les phospholipides et/ou leurs cofacteurs. Il peut être primaire ou secondaire à une maladie sous-jacente, principalement le lupus érythémateux systémique (LES) dans 15 à 30\% (El Amraoui et al., 2016 ; Cervera et al., 1993.). Le risque majeur est la survenue des événements thrombotiques responsable de diverses complications parmi lesquelles les complications obstétricales dont la fausse couche récurrente domine avec une fréquence entre 10 à 15\% (Rai et al., 1995 ; Yetman et al., 1996.). Malgré cette fréquence et son potentiel risque sur le foetus, le SAPL reste une affection rarement évoquée en première intention dans notre contexte au cours d'investigation d'une fausse couche si bien que le diagnostic est souvent égaré vers d'autres causes retardant ainsi la prise en charge. Ce travail montre le cas d'une femme de 36 ans, chez qui le diagnostic du SAPL a été fait tardivement après le $5^{\mathrm{e}}$ épisode de fausse couche. La $6^{\mathrm{e}}$ grossesse était conduite avec succès après un traitement incluant l'aspirine, corticoïdes et l'héparine. Ce qui motive à ajouter ce cas à ceux rapportés dans la littérature afin de susciter les praticiens à l'évoquer systématiquement au cours de la recherche étiologique d'une fausse couche.

\section{Presentation de Cas}

Madame T.N, âgée de 36 ans, aux antécédents d'ulcère de la jambe gauche, était adressée dans notre service pour l'exploration d'une pancytopénie. L'anamnèse retrouvait un début progressif de symptomatologie qui remontait à environ 10 mois par des fausses couches à répétition, totalisant cinq épisodes. Les deux premières fausses couches étaient survenues à partir de la $12^{\mathrm{e}}$ semaine d'aménorrhée sans suivi médical particulier. Les trois dernières grossesses étaient suivies au service de Gynécologie obstétrical ou trois cerclages ont été réalisés avec échec. Les fausses couches étaient survenues respectivement à partir de 8è, 10è et 11è semaine d'aménorrhée. Un mois après le dernier épisode de fausse couche s'était installée une asthénie physique, une palpitation, des vertiges, une tachycardie et une douleur des mollets. La patiente avait consulté à nouveau le service de Gynéco-obstétrique ou l'investigation avait retrouvé une pancytopénie associant une leucopénie à $0,7 \mathrm{G} / \mathrm{L}$, une anémie sévère à $4 \mathrm{~g} / \mathrm{dl}$ et une thrombopénie à $50 \mathrm{G} / \mathrm{L}$. La patiente avait bénéficié des séances de transfusion en concentré érythrocytaire 
successive totalisant $5000 \mathrm{ml}$ en (01) mois d'intervalle. Devant l'inefficacité transfusionnelle, elle nous a été adressée pour une exploration. Au plan clinique, l'examen retrouvait une patiente consciente et orientée avec l'indice performons de $1^{\prime} \mathrm{OMS}$ à 2 . Elle présentait une fièvre à $38^{\circ} \mathrm{C}$. Ses conjonctives étaient pâles et on notait un ictère sous conjonctivale et sclérotique. Il n'y avait pas des adénopathies à la palpation des aires ganglionnaires périphériques ni d'hépatomégalie. Par contre on avait retrouvé une splénomégalie type I selon la classification de Hacket. Au niveau de la peau, on notait des purpuras nodulaires diffus sur tout le corps. Il y'avait un gonflement du mollet droit, sensible avec diminution du ballottement. La dorsi-flexion du pied était très sensible. Le signe de Homans était positif. Les autres examens explorant le cœur, les poumons et les autres organes étaient sans particularité. Au plan biologique, l'hémogramme confirmait une pancytopénie sévère constituée des Globules Blancs à $0,32 \mathrm{G} / \mathrm{L}$; PNN à $0,16 \mathrm{G} / \mathrm{L}$, le taux d'Hémoglobine à $3 \mathrm{~g} / \mathrm{dl}$, VGM=85 fl, CCHM=32 pg et le nombre des plaquettes à $22 \mathrm{G} / \mathrm{L}$. nous avions réalisé un myélogramme qui retrouvait une moelle riche avec quelques anomalies morphologiques érythroblastiques, orientant la pancytopénie d'une cause périphérique car il n'y avait pas aussi des blastes. Nous avons donc réalisé un bilan immunologique de routine à s'avoir le test de coombs direct qui était revenu positif. Ce qui nous à motivé à évoquer une pancytopénie d'origine auto-immune. Nous avions poursuivi l'exploration par le dosage des anticorps antiphospholipides qui avait noté un titrage significatif. Le dosage des anticorps anti nucléaires était élevé à 640 UI/L avec une spécifié antiDNA natif. Par ailleurs, nous avions réalisé un echodopler du membre inferieur droit qui objectivait une thrombose des veines poplitées. Devant le contexte de fausse couche à répétition, la cytopénie auto-immune, la présence d'une thrombose des membres inferieurs et surtout le titrage significatif des anticorps anti phospholipides et des anticorps anti nucléaires, nous avions évoqué le diagnostic d'un syndrome des anticorps anti phospholipides lupiques. Nous avions instauré une corticothérapie en bolus avec le solumedrol (Méthyle prednisolone) $120 \mathrm{mg} /$ jour relayé par voie orale 5 jours après à dose de $1,5 \mathrm{mg} /$ jour associé à un immunosuppresseur par la cyclophosphamide $1 \mathrm{~g} / \mathrm{m}^{2} /$ mois en intraveineuse lente. Ce traitement était associé à un anti-thrombotique à base de l'aspirine $2 \mathrm{~g} /$ jour. Le traitement du lupus était aussi associé par le service de la rhumatologie à base de L'hydroxychloroquine (Plaquenil) 400mg/jour. L'évolution clinique était marquée par la régression progressive suivie de la disparition des signes cliniques et la normalisation de l'hémogramme en cinq (05) semaines. A l'annonce d'une 6è grossesse, 12 mois après, le reste du traitement était conduit sous l'aspirine et l'héparine de bas poids moléculaire. Ceci conduisait la grossesse avec succès au service de gynécologie obstétricale du CHU de Yopougon par la naissance d'un nouveau-né vivant. 


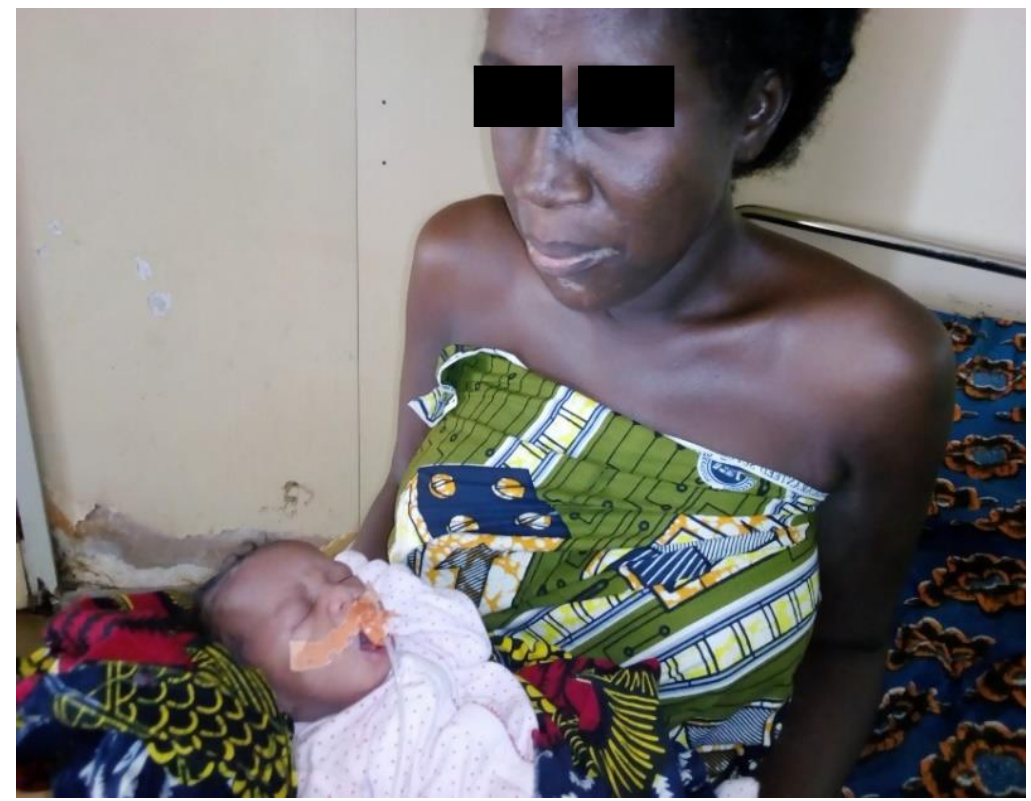

Fig 1 : patiente et le nouveau-né après le traitement

\section{Discussion}

Décrit par Graham Hugues en 1983 comme une affection thrombotique, le SAPL est caractérisé par la présence des anticorps antiphospholipide (aPL) dans le plasma associé à des signes cliniques de thromboses et des fausses-couches à répétition (Cervera et al., 1996 ; Di Prima et al., 2011). La prévalence des aPL dans la population saine est estimée entre 1 à 5,6\% et semble augmentée avec l'âge (De Groot et al., 2005 ; Richaud-Patin et al., 2000). L'âge moyen du diagnostic de SAPL est de 42 ans avec une prédominance féminine (Hanly, 2003). Cette tranche d'âge était proche de celle de la patiente dans ce travail qui avait 36 ans. Les signes cliniques et biologiques de SAPL sont polymorphes. Les atteintes sont en général systémiques et sont d'ordre hématologique, neurologique, obstétrical et dermatologique retrouvé dans le tableau I (Bonnie et al., 2008 ; Rogers, 2004). Ces signes étaient retrouvés chez cette patiente. Les signes hématologiques étaient la pancytopénie qui était d'ailleurs le principal motif de consultation dans le service d'Hématologie Clinique. Cette pancytopénie était autoimmune. La preuve était la positivité du test de coombs direct. Ce qui témoigne le caractère auto-immun du SAPL et du LES décrit par Cervera, Khamashta, Font, Sebastiani, Gil, Lavilla, Doménech, Aydintug et Jedryka-Góral (1993). Cependant, la profondeur cette cytopénie dans cette observation, avec un contexte de polytransfusion pourrait être du fait de son association avec le LES car l'association du SAPL avec ce dernier est un facteur de gravité (Cervera et al, 1993 ; Ruiz-Irastorza et al., 2004). Dans ce travaille, la patiente présentait aussi les signes thrombotiques. Les signes de Homans qui signe une 
thrombose des membres inférieurs était positive. D'ailleurs l'écho-dopler confirmait une thrombose de la veine poplitée. Cette patiente présentait aussi des signes cutanés. Il s'agissait des purpuras nécrotiques et l'ulcère de la jambe retrouvéàl'anamnèse. Wilson, Gharavi, Koike, Lockshin, Branch, Piette, Brey, Derksen, Harris, Hughes, Triplett et Khamashta (1999) ont décrit les signes dermatologiques du SAPL comme étant polymorphes et rapportaient la livedo reticularis comme étant le plus fréquent avec une fréquence de $40 \%$ bien que d'autres signes peuvent être observés. Il existait chez cette patiente, la notion de fausses couches récurrentes, un des éléments majeurs définissant le SAPL (Pengo et al., 2009). Delesalle, De Vienne, Le Hello, Verspyck et Dreyfus (2015) ont rapportés que les fausses couches au cours du SAPL surviennent généralement avant le 10è semaine. Chez la patiente dans ce travail, les deux premières fausses couches étaient survenues à partir de 12 Semaine d'aménorrhée et les trois autres avant le 10è semaine d'aménorrhée. Au point diagnostic, du fait de du caractère systémique et du polymorphisme clinique du SAPL, il existe plusieurs critères faisant son diagnostic. Le diagnostic actuel repose sur les critères de Sapporo établis en 1999 et révisés en 2006 (tableau II) (Pengo et al., 2009). Le titrage élevé des aPA, des anticorps anti-nucléaires et les données cliniques de la patiente de ce travail ont permis de retenir le diagnostic du SAPL lupique en tenant compte des critères diagnostiques de Sapporo (Pengo et al., 2009).

Tableau I : Manifestations cliniques du SAPL

(Bonnie et al., 2008 ; Rogers, 2004 ; Wilson et al., 1999).

\begin{tabular}{l} 
Thromboses artérielles et/ou veineuses \\
\hline Hématologiques \\
Cytopénie : thrombocytopénie, anémie hémolytique auto-immune, leucopénie \\
Saignement : dysfonction plaquettaire, déficit en prothrombine \\
Micro angiopathie thrombotique \\
Neurologiques \\
Ischémie aiguë (accident vasculaire cérébral, accident ischémique transitoire, \\
encéphalopathie), amnésie totale ou transitoire, lésions de la substance blanche \\
Obstétricales \\
Fausses couches spontanées à répétition, prématurité, pré-éclampsie \\
Cardiaques \\
Endocardite thrombotique non bactérienne, épaississement valvulaire, thrombus \\
intra cardiaque, épanchement péricardique, sténose prématurée \\
de greffon veineux de pontage \\
Pulmonaires \\
Embolie pulmonaire, HTAP, hémorragie alvéolaire \\
Cutanées \\
Livedo réticulaire, gangrène digitale, fissures hémorragiques, thrombose veineuse \\
superficielle, nécrose et infarctus, ulcérations \\
Autres manifestations \\
Thromboses rénales
\end{tabular}


Ischémie gastro-intestinale, nécrose et perforation de l'oesophage, ulcère duodénal atypique

Amaurose fugace, occlusion artérielle ou veineuse rétinienne, neuropathie optique antérieure

Insuffisance surrénalienne

Ostéonécrose de la tête fémorale

\section{SAPL catastrophique}

Evènements thrombotiques récurrents

Tableau II: critères diagnostiques de Sapporo (Pengo et al., 2009)

\section{Critères cliniques}

1) Thrombose(s) artérielle(s) et/ou veineuse(s) et/ou microvasculaire(s)

Un ou plusieurs épisodes de thrombose dans n'importe quel tissu ou organe. La thrombose doit être confirmée par l'imagerie ou par les données histopathologiques. Dans ce cas, il ne doit pas y avoir de signes histologiques de vascularite.

2) Événements obstétricaux

a) au moins une mort foetale (dès 10 semaines d'aménorrhée) inexpliquée par ailleurs,

b) ou une naissance prématurée (avant 34 semaines d'aménorrhée) d'un enfant morphologiquement normal, liée à une (pré)-éclampsie ou

une insuffisance placentaire sévère(s),

c) ou au moins trois avortements précoces (avant 10 semaines d'aménorrhée) consécutifs inexpliqués par ailleurs.

Critères biologiques (avec confirmation au-delà de 6 semaines)

1) Anticorps anticardiolipine IgG ou IgM à titre moyen ou élevé, par un test ELISA standardisé pour la recherche d'anticorps

dépendants de la b2GP1.

2) Anticoagulant circulant lupique dépisté selon les recommandations de l'International Society on Thrombosis and Hemostasis.

Le SAPL est " défini " s'il existe au moins un critère clinique et un critère biologique.

$\mathrm{Au}$ plan thérapeutique, l'association des corticoïdes, l'aspirine et l'héparine semblait entrainer une évolution favorable. Une cohorte historique de 11 ans sur le suivi des grossesses de 23 patientes diagnostiquées SAPL, Delasse et al (2015) avaient obtenu $87 \%$ de grossesses ayant abouties à la naissance des enfants vivants chez ces patientes traitées avec l'aspirine et l'héparine. Dans une autre étude menée par Le Guern et Goffinet (2008), le taux de grossesse conduit avec succès étaient de $70 \%$ sous ce même traitement au cours de SAPL. Ces études montrent que l'association d'un anticoagulant avec l'héparine améliorait le pronostic obstétrical des patientes. Les corticoides peuvent être associés en cas d'association au LES avec des cytopénies sévères. 


\section{Conclusion}

Le diagnostic du SAPL est vital en raison du risque thrombotique et des avortements récurrents. Cette observation devait susciter les praticiens à l'évoquer devant toutes fauches couches et les tests immunologiques doivent être pratiqués systématiquement dans ce contexte car la prise en charge ne devait pas être retardée.

\section{Conflit d'interet}

Les auteurs déclarent qu'ils n'ont pas de relation(s) personnelle (s) ou financière (s) qui peuvent avoir à tort influencé la rédaction de cet article.

\section{Contribution des Auteurs}

Dr N'DHATZ Comoe Emeraude et Dr PACKO Dieu-le-veut SaintCyr Sylvestre étaient les investigateurs principaux et ont effectué la recherche documentaire, le suivi de la patiente au service d'hématologie clinique et la rédaction de l'article. Professeur NANHO DANHO Clotaire, a participé à la prise en charge de la patiente et à la recherche bibliographique. Dr KARAMA Issa a suivi la patiente au service de Gynécologie et Obstétrique. Professeur MBELESSO Pascal (Chef de service de Neurologie, CHU de l'amitié SinoCentrafricaine) et Dr DIAKITE Mamady (chef de service d'Hématologie Clinique de l'Hôpital National de Donka, Guinée) ont révisé l'article. Professeur Koffi Kouassi Gustave a participé à l'encadrement scientifique de ce travail.

\section{Remerciements}

Nos sincères remerciements à toutes les équipes du service d'Hématologie Clinique et Gynécologie Obstétrique du CHU de Yopougon qui ont contribuées à la rédaction de cet article et à la prise en charge de cette patiente. Nous saluons aussi la parfaite collaboration de cette patiente au cours de son suivi.

\section{References :}

1. Bonnie, LB., Doruk, E., \& Peter, HS. (2008). Clinical manifestations of the antiphospholipid syndrome. www.uptodate.com.

2. Cervera, R., Khamashta, MA., Font, JO., Sebastiani, GD., Gil, AN., Lavilla, P., Doménech, I., Aydintug, AO., \& Jedryka-Góral, AN. (1993). Systemic lupus erythematosus: clinical and immunologic patterns of disease expression in a cohort of 1,000 patients. The European Working Party on Systemic Lupus Erythematosus. Medicine; 72(2):113-24.

3. Di Prima, FAF., Valenti, O., Hyseni, E., Giorgio, E., Faraci, M., Renda, E., De Domenico, R., \& Monte, S. (2011). 
Antiphospholipid Syndrome during pregnancy: the state of the art. J Prenat Med; 5(2): 41-53.

4. De Groot, PG., Lutters, B., Derksen, R., Lisman, T., Meijers, JC., \& Sendaal, FR. (2005). Lupus anticoagulants and the risk of a first episode of deep venous thrombosis. J Thromb Haemost ;3:1993-7.

5. Delesalle, C., de Vienne, C., Le Hello, C., Verspyck, E., \& Dreyfus, M. (2015). Antiphospholipid syndrome and pregnancy: obstetrical prognosis according to the type of APS. J Gynecol Obstet Biol Reprod (Paris);44(5):463-70.

6. El Amraoui, M., Bounnyit, H., Zemmez, Y., Bouhamidi, A., Hassam, B., \& Senouci, K. (2016). Nécroses cutanées multiples et extensives révélant un syndrome des anticorps anti phospholipids. The Pan Afr Med J;25:56.

7. Hanly, JG. (2003). Antiphospholipid syndrom: an overview. CMAJ; 168 (13):1675-82

8. Le Guern, V. \& Goffinet, F. (2008). Grossesse et anticorps antiphospholipides. Presse Méd ; 37 :1666-75.

9. Pengo, V., Tripodi, A., Reber, G., Rand, JH., Ortel, TL., Galli, M., \& De Groot, PG. (2009). Update of the guidelines for lupus anticoagulant detection. Subcommittee on lupus anticoagulant/antiphospholipid antibody of the scientific and standardisation committee of the International Society on Thrombosis and Haemostasis. J Thromb Haemost; 7:1737-40.

10. Rai, RS., Regan, L., Clifford, K., Pickering, W., Dave, M., Mackie, I., McNally, T., \& Cohen, H. (1995). Immunology: Antiphospholipid antibodies and $\beta 2$-glycoprotein-I in 500 women with recurrent miscarriage: results of a comprehensive screening approach. Hum Reprod; 10(8):2001-5.

11. Richaud-Patin, Y., Cabiedes, J., Jakez-Ocampo, J., Vidaller, A., \& Llorente, L. (2000). High prevalence of protein-dependent and proteinindependent antiphospholipid. Thromb. Res ; 99:129-33.

12. Ruiz-Irastorza, G., Egurbide, MV., Ugalde, J., \& Aguirre, C. (2004). High impact of antiphospholipid syndrome on irreversible organ damage and survival of patients with systemic lupus erythematosus. Arch Intern Med.; 164:77-82.

13. Rogers, G.M. (2004). Acquired Coagulation Disorders.Antiphospholipid-Protein Antibodies: Lupus Anticoagulants and Anticardiolipin Antibodies.pp1699- 1700; inWindrobe's clinical Hematology 11 ième ED., Lippincott Williams and Wilkins Eds.

14. Wilson, WA., Gharavi, AE., Koike, T., Lockshin, MD., Branch, DW., Piette, JC., Brey, R., Derksen, R., Harris, EN., Hughes, 
GR., Triplett, DA., \& Khamashta, MA. (1999). International consensus statement on preliminary classification criteria for definite antiphospholipid syndrome: report of an international workshop.Arthritis Rheum; 42: 1309-11.

15. Yetman, DL. \& Kutteh, WH. (1996). Antiphospholipid antibody panels and recurrent pregnancy loss: prevalence of anticardiolipin antibodies compared with other antiphospholipid antibodies. Fertil Steril; 66:540-6. 